\title{
BMJ Open Risk factors associated with purchasing pesticide from shops for self-poisoning: a protocol for a population-based case-control study
}

\author{
Manjula Weerasinghe, ${ }^{1,2}$ Flemming Konradsen, ${ }^{2,3}$ Michael Eddleston, ${ }^{2,3,4}$ \\ Melissa Pearson, ${ }^{2,4}$ David Gunnell, ${ }^{5}$ Keith Hawton, ${ }^{6}$ Shaluka Jayamanne, ${ }^{2,7}$ \\ Chathurani Pabasara, ${ }^{2}$ Tharidu Jayathilaka, ${ }^{2}$ Kalpani Dissanayaka, ${ }^{2}$ \\ Sandamali Rajapaksha, ${ }^{2}$ Prasanna Thilakarathna, ${ }^{2}$ Suneth Agampodi ${ }^{1}$
}

To cite: Weerasinghe $M$, Konradsen F, Eddleston M, et al. Risk factors associated with purchasing pesticide from shops for selfpoisoning: a protocol for a population-based case-control study. BMJ Open 2015;5:e007822. doi:10.1136/bmjopen-2015007822

- Prepublication history for this paper is available online. To view these files please visit the journal online (http://dx.doi.org/10.1136/ bmjopen-2015-007822).

Received 29 January 2015 Accepted 2 March 2015

CrossMark

For numbered affiliations see end of article.

Correspondence to Professor Michael Eddleston; eddlestonm@yahoo.com

\section{ABSTRACT}

Introduction: Pesticide self-poisoning is one of the most frequently used methods of suicide worldwide, killing over 300000 people annually. Around $15-20 \%$ of pesticide self-poisonings occur soon after the person has bought the pesticide from a shop. We aim to determine the characteristics of individuals who purchase pesticides directly from shops and how they differ from individuals who access pesticides from other sources such as home, home garden or farmland. This information will help inform possible vendor/shop-based intervention strategies aimed at reducing access to pesticides used for self-harm.

Methods and analysis: This study will investigate risk factors associated with purchasing pesticides for acts of self-poisoning from pesticide shops, including cases identified over a 9-month period using a populationbased case-control group approach. Four intervieweradministered data collection tools will be used for this study: a semistructured questionnaire, Beck Suicidal Intent Scale (SIS), Clinical Interview ScheduleSinhalese version (CIS-Sn) and Alcohol Use Disorders Identification Test (AUDIT). Each case (expected $n=33$ ) will be compared with two groups of individuals: (1) those who have self-poisoned using pesticides from the home, home garden or farmland and (2) those who bought pesticides from the same shops as the above cases, but not did not self-poison. Logistic regression models will be used to identify risk factors of purchasing pesticides for self-poisoning from shops.

Ethics and dissemination: The study has received ethical approval from the Ethical Review Committee of the Faculty of Medicine and Allied Sciences, Rajarata University of Sri Lanka. A sensitive data collection technique will be used and ethical issues will be considered throughout the study. Results will be disseminated in scientific peer-reviewed articles.

\section{INTRODUCTION}

Worldwide, the WHO now considers pesticides and hanging to be the most frequently

\section{Strengths and limitations of this study}

- No published data are available on factors that may help identify individuals purchasing pesticide from shops for the purpose of selfpoisoning; the results of this study will be the first step in developing interventions to reduce the incidence of such episodes.

- The major limitation of the study is the inability to interview people who died following selfpoisoning. In addition, the sensitivity of the issues discussed with interviewees may have an impact on the willingness of individuals to participate in the study.

- Cases and controls will be identified from selfreported information.

used methods of suicide; ${ }^{1}$ pesticides alone are responsible for around 1/3 of global suicides. $^{2}{ }^{3}$ Pesticide self-poisoning is more common in rural Asia than in Western countries. ${ }^{4}$ Case-fatality following pesticide poisoning is over 20 times higher than that of poisoning with medicines-the commonest method of hospital presenting self-harm in the West. ${ }^{5}$ Such differences may account for the disproportionately high rates of suicide in rural Asia.

Easy availability of pesticides in the domestic environment of farming households ${ }^{6}$ is believed to be responsible for pesticides being the most common means of self-harm in Sri Lanka. ${ }^{7}$ Around $15-20 \%$ of pesticide self-poisonings occur soon after the person has bought the pesticide from a shop with the intention of drinking it. ${ }^{8-10}$ Our previous work with pesticide vendors indicated that they are acutely aware of the problem and of deaths that have occurred following purchase of pesticides at their shop. ${ }^{7}$ Although neither the pesticide vendors nor the community 
consider the vendors responsible, the vendors are keen to find ways to reduce the number of times self-harm occurs soon after purchase of pesticides. ${ }^{7}$ Thus far, no research has taken place to determine whether interventions implemented through pesticide shops might be a suitable means for prevention.

Restricting pesticide availability to prevent their use for impulsive self-harm is likely to be an effective means to reduce deaths from self-poisoning. ${ }^{11-14}$ A wide range of interventions have been tested to prevent suicide by reducing access to means at the point of sale. These include analgesic packaging restrictions $;{ }^{15}{ }^{16}$ restrictions on gun sales, including waiting times before purchase, age limits and licenses; ${ }^{17-21}$ physical barriers to purchases of charcoal ${ }^{22}$ and gatekeeper training. ${ }^{13}$ Such approaches could be extended to pesticide availability from retail outlets. There is convincing evidence that restricting access to commonly-used highly lethal methods of suicide not only reduces method-specific suicide rates, but also reduces overall rates. ${ }^{23} 24$

In order to identify potential interventions implemented through pesticide shops, we will undertake a casecontrol study to determine the characteristics of individuals who purchase pesticides directly from shops and how they differ from individuals who access pesticides from other places, such as their home, home garden or farmlands. The results from the case-control study will be used to plan a community-based public health intervention at sales points to prevent pesticide selfpoisoning. An effective intervention could contribute to saving many lives every year across rural Asia.

The objectives of this study are:

1. To compare the factors associated with different pesticide access points, that is, purchasing a pesticide from a shop or accessing pesticides from home, home garden or farmlands for self-poisoning.

2. To determine individual and household level factors associated with purchase of pesticide from shops for self-poisoning as compared with purchases of pesticides not for the purpose of self-poisoning.

\section{METHODS AND ANALYSIS}

Study design

This study is a population-based case-control study to determine the factors at individual and household levels, and other selected factors associated with purchasing pesticides from shops for the purpose of self-poisoning. Individuals who bought pesticides specifically for the act of self-poisoning will be identified; these cases will be compared with two sets of unmatched controls;

1. Control group A-those who accessed pesticides from home, home garden or farm lands for selfpoisoning. Up to four will be chosen at random (unmatched) for each case.

2. Control group B-those who bought pesticides for agricultural purposes at the same shop within seven days of purchase by the relevant case without using these for self-harm. Four will be chosen at random (unmatched) for each case.

\section{Study setting}

The study will be carried out in Anuradhapura District, North Central Province of Sri Lanka. The main study area (figure 1) comprises 162 Grama Niladari divisions (GN, the lowest administrative division) located in 6 divisional secretariats (DS, a government administrative region with population of about 40000 people) of Thambuttegama, Thalawa, Nochchiyagama, Rajanganaya, Galnewa and Ipologama with a total population of 256376 inhabitants (Census 2011). The study area is located in the dry zone of central Sri Lanka, receives irrigated water as part of the Mahaweli $\mathrm{H}$ and Rajanganaya irrigation systems, has at least two cropping seasons per year, and the majority of farmers are involved in irrigated paddy, vegetable and banana cultivation. The use of agricultural pesticides is high in this area. There is an estimated rate of 315 to 447 per 100000 population who attempt suicide by all means of self-poisoning. ${ }^{48}{ }^{25}$ In the study area, Sinhalese Buddhists are the majority religious group.

\section{Participants and recruitment}

Cases and Control group A will be selected from the ongoing Safe Storage prospective cohort $^{26}$ identified through the rural hospital network. This consists of 11 peripheral hospitals and 2 secondary/tertiary care hospitals. These hospitals care for $>99 \%$ of self-harm admissions (unpublished data from Safe Storage $s t u d y^{26}$ ) from the study area.

Control group B participants are those who bought pesticides at the same shop within 7 days of the relevant case purchase without using these for self-harm. Four control group B participants will be chosen at random from among a pool of potentially eligible controls identified at the pesticide shop from which the pesticides were purchased for self-harm.

\section{Inclusion and exclusion criteria}

All non-fatal cases of agricultural pesticide self-poisoning who are admitted to one of the 13 study hospitals from the defined study area over the 9-month period will be eligible for study entry as cases and control group A. The participants will be either permanent residents or people who were temporary residents in the study area at the time of the incident. All pesticide shops, regardless of whether or not they hold a government license to sell pesticides, will be included in the study. However, pesticide shops located $20 \mathrm{~km}$ or more from the study area boundary will be excluded from the study because of logistic issues. The minimum age limit for the participants is 16 years and there is no upper age limit. The age of 16 was identified to ensure the study would capture adolescent self-poisoning attempts, especially among young girls among whom the prevalence is highest in the $16-19$ years age group. ${ }^{27-29}$ 


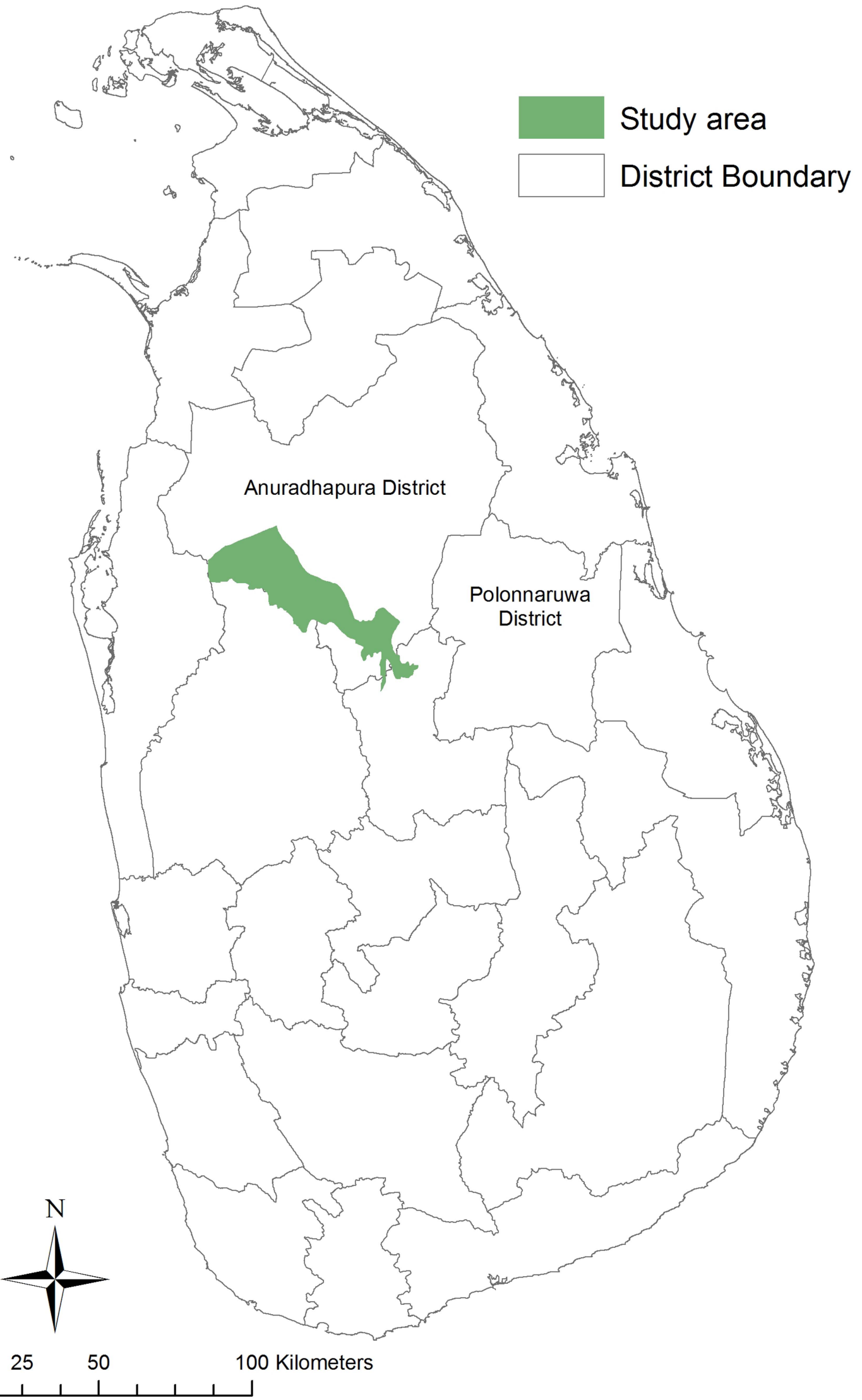

Figure 1 The study region to the south west of the Anuradhapura District, Sri Lanka. 
Deaf patients and patients who are critically ill and unable to recall the self-poisoning incident will be excluded from the study. Fatal self-poisoned patients will also be excluded from the study as data obtained from next of kin are likely to be inaccurate or missing important study variables. Participants who are not confident of being interviewed in Sinhala language will be excluded from the study (based on unpublished data from the Safe Storage study ${ }^{26}$ we expect that $<1 \%$ of the patients cannot be interviewed in Sinhala).

\section{Data collection}

The study is expected to recruit participants over a 9-month period. Before the start of the data collection, study instruments will be pretested on 20 cases of pesticide self-poisoning and 20 shop controls (control group B) from outside of the study area. We will finalise our interviews and questionnaires based on experience from the pilot interviews.

Each individual selected as a case or control group A participant will be interviewed initially at the hospital to obtain basic information, including name and address; an explanation of the study will be given and their permission sought to participate in it. If the patient agrees, they will be contacted by the team and an appointment made to visit the participant at home within 7 days of hospital discharge.

Each customer identified as a control group B participant will be interviewed initially at the pesticide shop just after the purchase to obtain basic information, including name and address; an explanation of the study will be given and their permission sought to participate in it. The participants will then be interviewed in their households within 7 days of initial identification.

The researchers will work in pairs to ensure similar recording of data and to maximise inter-rater reliability. Interviews will be conducted in the local language (Sinhala) in the participants' household. As many of the questions are sensitive in nature, the main interviews will conducted when the patients are discharged from the hospital and are back in their home environment. If individuals are initially not approachable because of their medical conditions, then the most suitable time will be chosen for the interviews. Interviews will be conducted with each participant individually, away from other family or community members to increase the likelihood that their responses will not be influenced by any other person.

\section{Questionnaire}

An interviewer-administered semistructured questionnaire will be used to record risk factors associated with purchasing pesticides from shops. The questionnaire is formulated in English and translated into Sinhala. The questionnaire mainly consisted of closed questions but also included some multiple choice questions with options being mutually exclusive and semiopen questions. Questions and possible answers are precoded with numerical codes to facilitate entry into the computerised database. The questionnaire is used to elicit sociodemographic, ingested pesticide information and history of self-harm. For those patients defined as cases and control group A participants, additional questions are included regarding the poisoning event and what happened before, during and after the purchase.

In addition to the questionnaire, three validated measures of suicide intent, mental health and alcohol misuse will be applied: the Beck Suicidal Intent Scale (SIS) ${ }^{30}$ the Clinical Interview Schedule-Sinhalese version ${ }^{31}$ (CIS-Sn), and the Alcohol Use Disorders Identification Test $^{32}$ (AUDIT).

\section{The Beck Suicidal Intent Scale}

SIS is a 15-item questionnaire designed to assess the severity of suicidal intent associated with an episode of self-harm. ${ }^{30}$ Each item is scored 0-2, giving a total score range of $0-30$. The final score $<10,10-15$ and $>15$ will be taken as low intent, medium intent and high intent, respectively. ${ }^{33}$ This tool has been translated into Sinhala and currently being used in a community-based study (unpublished).

\section{Clinical Interview Schedule-Sinhalese version}

The CIS-R has been widely used in the assessment of common mental disorders. ${ }^{34}$ The CIS-Sn is a slightly modified version of CIS-R; CIS-Sn was validated in a clinical setting for adolescents 15-19 years of age in Sri Lanka. ${ }^{31}$ The CIS-Sn includes sections introducing each symptom group: fatigue, lack of concentration and forgetfulness, irritability, worry about physical health, depression, depressive ideas, worry, anxiety, panic, compulsions and obsessions. CIS-Sn section scores of $\geq 2$ are taken as positive for the symptom group.

\section{The Alcohol Use Disorders Identification Test}

AUDIT is unique among alcohol-related screening instruments in that it is designed to measure a range of risk levels, from low risk drinking to hazardous drinking and alcohol use disorders. AUDIT is a 10 -item scale which identifies the level of alcohol use. Score of $\geq 8$ is indicative of hazardous drinking and scores of $\geq 20$ is suggestive of alcohol dependence. It has been translated into Sinhala and validated, ${ }^{32}$ and has been previously used on a military population in Sri Lanka. ${ }^{35}$

\section{Sample size calculation}

The estimated annual incidence of hospital admission for pesticide self-poisoning within the study area is 175 cases per 100000 (unpublished data from the Safe Storage $\left.s t u d y^{26}\right)$. Within the study population of 200000 , we expect at least 292 admissions each year for pesticide self-poisoning (this conservative number is based on the Safe Storage intervention being highly effective; therefore, $175 / 100000$ in the Safe Storage control area and 117/100 000 in the intervention area). If $15 \%$ of these cases followed direct purchase of the 
pesticide from a vendor, we expect 44 cases to occur annually (or 33 cases over the 9-month study period). Four controls matched for age, gender and village will be selected for each case from Safe Storage cases accessing pesticides stored within the house or home garden. This sample size provides $80 \%$ power (at the $5 \%$ level of statistical significance) to detect risk ratios of 2.5 or more in relation to risk factors with a $50 \%$ prevalence in the control group.

\section{Analysis plan}

Ordinary logistic regression models will be used to investigate associations of the following potential risk factors with purchasing pesticides for the purpose of self-harm from a pesticide shop. Potential to be examined include: patient demographics, in particular their age and sex, number of years in completed education, whether respondent is a farmer, whether the respondent has previous experiences of buying pesticides, mode of purchase (cash or credit), purchasing one pesticide bottle, purchasing a liquid pesticide bottle, events preceding incident including interval from purchase to incident, time and week day of purchase; proximity to a national holiday, amount and cost of poison purchased, familiarity of purchaser with the vendor, whether the person was under the influence of alcohol at the time of purchase and the self-harm history.

The study will investigate the potential confounding effects of age, sex and socioeconomic status as these individual factors may influence the chosen method of access. Differences between cases and controls for confounding factors will be tested using $\chi^{2}$ statistics. Multiple logistic regression will be used to adjust for confounding by age, sex and socioeconomic status to obtain adjusted estimates of ORs and 95\% CIs for the independent effects of the variables of interest. The Statistical Package for Social Science (SPSS) by SPSS Inc, Chicago, Illinois, USA, V.22.0 will be used for data analysis.

\section{Potential impact and future work}

This study will identify risk factors for the purchase of pesticides just prior to and for the purpose of self-harm. This information will be used to identify interventions that may be effective in preventing pesticide self-harm through large-scale public health trials. One outcome for this study will be the design of a pilot intervention study.

An effective intervention has the potential to stop around 15-20\% of acts of pesticide self-poisoning. Evidence for prevention of this form of self-poisoning via interventions involving private vendors may also be useful for preventing medicine self-poisoning, a problem that is on the increase in Sri Lanka. ${ }^{28}$ Although deaths from medicine poisoning are much less common than from pesticide poisoning, overdoses with medicine is becoming a significant public health problem in Sri Lanka and may become more important as pesticide self-poisoning is controlled.

\section{DISSEMINATION}

The main risk of this study is that discussion concerning self-harm may cause distress. All individuals recruited will provide written informed consent to partake in the study. We work closely with Courage Compassion Commitment (CCC) foundation's helpline and a local, highly-regarded NGO, Sumithrayo, which works as a Samaritans/ Befrienders international organisation in our area. Their telephone number will be provided to all people in the study.

The research is unlikely to offer benefit to the individuals involved unless discussion of the events is therapeutic. However, potential interventions identified from the study offer major public health benefits to the community over the long run. These benefits have previously been identified by the local communities we have worked with over the past 15 or more years, resulting in very high rates of recruitment to both clinical and public health intervention studies.

The information gained will be directly relevant to about $15-20 \%$ of all pesticide suicides, an estimated 30 000-45 000 deaths every year across Asia. An effective intervention offers the possibility of saving many lives every year.

\section{Author affiliations}

${ }^{1}$ Department of Community Medicine, Faculty of Medicine \& Allied Sciences, Rajarata University of Sri Lanka, Saliyapura, Anuradhapura, Sri Lanka ${ }^{2}$ Faculty of Medicine, South Asian Clinical Toxicology Research Collaboration (SACTRC), University of Peradeniya, Peradeniya, Sri Lanka

${ }^{3}$ Department of Public Health, Faculty of Sciences, University of Copenhagen, Copenhagen, Denmark

${ }^{4}$ Pharmacology, Toxicology and Therapeutics, University of Edinburgh, Edinburgh, UK

${ }^{5}$ School of Social and Community Medicine, University of Bristol, Bristol, UK ${ }^{6}$ Department of Psychiatry, Centre for Suicide Research, University of Oxford, Oxford, UK

${ }^{7}$ Department of Medicine, Faculty of Medicine, University of Kelaniya, Ragama, Sri Lanka

Acknowledgements The authors would like to thank the Safe Storage field staff including Chaminda Prasad, Malith Herath, Iresha Palugaswewa, Arunasiri Muhandiram for their support in participant identification at hospitals and pesticide shops. The authors appreciate the management and organisational support from Umesh Chathuranga, Mala Ranawake and Indunil Abeyrathna. The authors thank the Provincial Ministries of Health and Agriculture, the Office of the Pesticide Registrar and hospital staffs for their support to set-up the study. DG and KH are both National Institute for Health Research (England) Senior Investigators.

Contributors MW drafted the initial manuscript and developed the protocol. SA, FK, ME, MP, DG, KH and SJ collaborated on conceptualising and designing the study and contributed to the draft of the manuscript. ME contributed towards the study set-up. SA contributed to ethical considerations. SA, FK and DG provided valuable inputs to methodology. MP contributed towards the study set-up, and logistics and methodology. MW, CP, KD, SR, TJ and PT implemented the study and the collecting of data, and contributed to the first draft. All authors read and approved the final manuscript.

Funding The work is supported by the American Foundation of Suicide Prevention (DIG-0-095-12).

Competing interests None.

Ethics approval Ethics approval was granted from the research, ethics and the higher degrees committee of Rajarata University, Sri Lanka, in October 2013. 
Approval for the study has been received from key policymakers in the Provincial Departments of Health Services and Agriculture of the North Central Province, the Office of the Registrar of Pesticides and the Pesticide Technical and Advisory Committee (PeTAC) of Sri Lanka.

Provenance and peer review Not commissioned; externally peer reviewed.

Open Access This is an Open Access article distributed in accordance with the Creative Commons Attribution Non Commercial (CC BY-NC 4.0) license, which permits others to distribute, remix, adapt, build upon this work noncommercially, and license their derivative works on different terms, provided the original work is properly cited and the use is non-commercial. See: http:// creativecommons.org/licenses/by-nc/4.0/

\section{REFERENCES}

1. World Health Organization. WHOIPreventing suicide: a global imperative. World Health Organization, 2014. http://www.who.int/ mental_health/suicide-prevention/world_report_2014/en/ (accessed 27 Dec 2014)

2. Bertolote JM, Fleischmann A, Eddleston M, et al. Deaths from pesticide poisoning: a global response. $\mathrm{Br} J$ Psychiatry 2006;189:201-3.

3. Bertolote JM, Fleischmann A, Butchart A, et al. Suicide, suicide attempts and pesticides: a major hidden public health problem. Bull World Health Organ 2006;84:260.

4. Eddleston M, Phillips MR. Self poisoning with pesticides. BMJ 2004;328:42-4.

5. Gunnell D, Eddleston M. Suicide by intentional ingestion of pesticides: a continuing tragedy in developing countries. Int $J$ Epidemiol 2003;32:902-9. http://www.pubmedcentral.nih.gov/ articlerender.fcgi?artid=2001280\&tool=pmcentrez\&rendertype= abstract (accessed 21 Mar 2014).

6. Konradsen F, van der Hoek W, Peiris P. Reaching for the bottle of pesticide-a cry for help. Self-inflicted poisonings in Sri Lanka. Soc Sci Med 2006;62:1710-19.

7. Weerasinghe $\mathrm{M}$, Pearson $\mathrm{M}$, Peiris $\mathrm{R}$, et al. The role of private pesticide vendors in preventing access to pesticides for self-poisoning in rural Sri Lanka. Inj Prev 2014;20:134-7.

8. Eddleston M, Karunaratne A, Weerakoon M, et al. Choice of poison for intentional self-poisoning in rural Sri Lanka. Clin Toxicol (Phila) 2006;44:283-6. http://www.pubmedcentral.nih.gov/articlerender.fcgi? artid=1940039\&tool=pmcentrez\&rendertype=abstract (accessed 21 Mar 2014)

9. Bose A, Sandal Sejbaek C, Suganthy P, et al. Self-harm and self-poisoning in southern India: choice of poisoning agents and treatment. Trop Med Int Health 2009;14:761-5.

10. Mohamed F, Manuweera G, Gunnell D, et al. Pattern of pesticide storage before pesticide self-poisoning in rural Sri Lanka. BMC Public Health 2009;9:405.

11. Goldney RD. Suicide prevention: a pragmatic review of recent studies. Crisis 2005;26:128-40. http://www.ncbi.nlm.nih.gov/ pubmed/16276756 (accessed 21 Mar 2014).

12. Manorantjtham S, Abraham S, Jacob KS. Towards a national strategy to reduce suicide in India. Natl Med $\mathrm{J}$ India 2005;18:118-22. http://www.ncbi.nlm.nih.gov/pubmed/16130610 (accessed 21 Mar 2014).

13. Mann JJ, Apter A, Bertolote J, et al. Suicide prevention strategies: a systematic review. JAMA 2005;294:2064-74.

14. Nordentoft M. Restrictions in availability of drugs used for suicide. Crisis 2007;28:44-9.

15. Hawton $\mathrm{K}$ Townsend $\mathrm{E}$ Deeks J, et al. Effects of legislation restricting pack sizes of paracetamol and salicylate on self poisoning in the United Kingdom: before and after study. BMJ 2001;322:1203-7.

16. Sheen $\mathrm{CL}$, Dillon JF, Bateman DN, et al. Paracetamol pack size restriction: the impact on paracetamol poisoning and the over-the-counter supply of paracetamol, aspirin and ibuprofen. Pharmacoepidemiol Drug Saf 2002;11:329-31.

17. Loftin C, McDowall D, Wiersema B, et al. Effects of restrictive licensing of handguns on homicide and suicide in the District of Columbia. N Engl J Med 1991;325:1615-20.

18. Miller M, Lippmann SJ, Azrael D, et al. Household firearm ownership and rates of suicide across the 50 United States. J Trauma 2007;62:1029-34; discussion 1034-5.

19. Beautrais AL, Fergusson DM, Horwood LJ. Firearms legislation and reductions in firearm-related suicide deaths in New Zealand. Aust $N$ Z J Psychiatry 2006;40:253-9.

20. Kapusta ND, Etzersdorfer E, Krall C, et al. Firearm legislation reform in the European Union: impact on firearm availability, firearm suicide and homicide rates in Austria. Br J Psychiatry 2007;191:253-7.

21. Klieve H, Barnes M, De Leo D. Controlling firearms use in Australia: has the 1996 gun law reform produced the decrease in rates of suicide with this method? Soc Psychiatry Psychiatr Epidemiol 2009;44:285-92.

22. Yip PSF, Law CK, Fu et al. Restricting the means of suicide by charcoal burning. Br J Psychiatry 2010;196:241-2.

23. Gunnell D, Fernando R, Hewagama M, et al. The impact of pesticide regulations on suicide in Sri Lanka. Int $J$ Epidemiol 2007;36:1235-42.

24. Hawton K. Restricting access to methods of suicide: rationale and evaluation of this approach to suicide prevention.Crisis 2007;28:4-9.

25. Dawson $\mathrm{AH}$, Eddleston $\mathrm{M}$, Senarathna $\mathrm{L}$, et al. Acute human lethal toxicity of agricultural pesticides: a prospective cohort study. PLoS Med 2010;7:e1000357.

26. Pearson M, Konradsen F, Gunnell D, et al. A community-based cluster randomised trial of safe storage to reduce pesticide self-poisoning in rural Sri Lanka: study protocol. BMC Public Health 2011;11:879.

27. Senarathna L, Jayamanna SF, Kelly PJ, et al. Changing epidemiologic patterns of deliberate self poisoning in a rural district of Sri Lanka. BMC Public Health 2012;12:593.

28. Marecek J, Senadheera C. "I drank it to put an end to me": Narrating girls' suicide and self-harm in Sri Lanka. Contrib Indian Soc 2012;46:53-82.

29. Senadheera C, Marecek J, Hewage C, et al. A hospital-based study on trends in deliberate self-harm in children and adolescents. Ceylon Med J 2010;55:67-8. http://www.ncbi.nlm.nih.gov/pubmed/20645550 (accessed 1 Jan 2015)

30. Beck AT, Schuyler D, Herman I. Development of suicidal intent scales. 1974.

31. Wickramasinghe SC, Rajapakse L, Abeysinghe R, et al. The Clinical Interview Schedule-Sinhala version: validation in a community setting in Sri Lanka. Int J Methods Psychiatr Res 2002;11:169-77. http://www.ncbi.nlm.nih.gov/pubmed/12459820 (accessed 24 Apr 2014).

32. De Silva $P$, Jayawardana $P$, Pathmeswaran A. Concurrent validity of the alcohol use disorders identification test (AUDIT). Alcohol Alcoho 2008:43:49-50

33. Conner KR, Phillips MR, Meldrum SC. Predictors of low-intent and high-intent suicide attempts in rural China. Am J Public Health 2007:97:1842-6.

34. Lewis G, Pelosi AJ, Araya R, et al. Measuring psychiatric disorder in the community: a standardized assessment for use by lay interviewers. Psychol Med 1992;22:465-86. http://journals.cambridge.org/abstract_ S0033291700030415 (accessed 19 Nov 2014)

35. Hanwella R, de Silva VA, Jayasekera NE. Alcohol use in a military population deployed in combat areas: a cross sectional study. Subst Abuse Treat Prev Policy 2012;7:24. http://www. substanceabusepolicy.com/content/pdf/1747-597X-7-24.pdf (accessed 27 Nov 2014). doi:10.1186/1747-597X-7-24

36. De Silva VA, Senanayake SM, Dias $\mathrm{P}$, et al. From pesticides to medicinal drugs: time series analyses of methods of self-harm in Sri Lanka. Bull World Health Organ 2012;90:40-6. 